\title{
A 3D Porous Media Liver Lobule Model: the Importance of Vascular Septa and Anisotropic Permeability for Homogeneous Perfusion
}

Charlotte Debbaut $^{1}$, Jan Vierendeels ${ }^{2}$, Jennifer H. Siggers ${ }^{3}$, Rodolfo Repetto $^{4}$, Diethard Monbaliu ${ }^{5}$ and Patrick Segers ${ }^{1}$

${ }^{1}$ Biofluid, Tissue and Solid Mechanics for Medical Applications (bioMMeda), Institute Biomedical Technology, Ghent University. Campus Heymans - Blok B, De Pintelaan 185, 9000 Gent, Belgium. Telephone: +3293313137.

Email: charlotte.debbaut@ugent.be (corresponding author), patrick.segers@ugent.be

${ }^{2}$ Department of Flow, Heat and Combustion Mechanics, Ghent University. Sint-

Pietersnieuwstraat 41, 9000, Gent, Belgium.

Telephone: +329264 33 13. Email: jan.vierendeels@ugent.be

${ }^{3}$ Department of Bioengineering, Imperial College London. London SW7 2AZ, United Kingdom.

Telephone: +44 (0)20 7594 3663. Email: j.siggers@imperial.ac.uk

${ }^{4}$ Department of Civil, Chemical and Environmental Engineering, and Center for

Material Science and Technology

University of Genova. Via Montallego 1, 16145 Genova, Italy.

Telephone: +39010 3532432. Email: rodolfo.repetto@unige.it

${ }^{5}$ Department of Abdominal Transplant Surgery, University Hospitals Leuven, Catholic University Leuven, Leuven, Belgium. Herestraat 49, 3000 Leuven, Belgium.

Telephone: +32 163487 27. Email: diethard.monbaliu@uzleuven.be 


\section{A 3D Porous Media Liver Lobule Model: the Importance of Vascular Septa and Anisotropic Permeability for Homogeneous Perfusion}

The hepatic blood circulation is complex, particularly at the microcirculatory level. Previously, 2D liver lobule models using porous media and a 3D model using real sinusoidal geometries have been developed. We extended these models to investigate the role of vascular septa (VS) and anisotropic permeability.

The lobule was modelled as a hexagonal prism (with or without VS) and the tissue treated as a porous medium (isotropic or anisotropic permeability). Models were solved using computational fluid dynamics.

VS inclusion resulted in more spatially homogeneous perfusion. Anisotropic permeability resulted in a larger axial velocity component than isotropic. A parameter study revealed that results are most sensitive to the lobule size and radial pressure drop.

Our model provides insight into hepatic microhemodynamics, and suggests that inclusion of VS in the model leads to perfusion patterns that are likely to reflect physiological reality. The model has potential for applications to unphysiological and pathological conditions.

Keywords: hepatic microcirculation, liver lobule, computational fluid dynamics, anisotropic permeability, porous medium, vascular septa 


\section{Introduction}

The perfusion of the liver is complex and distinct from that of other organs, mainly due to its dual blood supply from both the hepatic artery and the portal vein [1-4]. In particular, the microcirculation on the scale of the liver lobules and sinusoids displays complicated hemodynamics [2, 3, 5-9].

In the literature, microperfusion of the liver is often schematically represented in terms of classical lobules, each having the shape of a hexagonal prism (Fig. 1) [2, 3]. Liver lobules are assumed to be configured in a tessellating pattern, similar to the organisation of hexagonal wax cells in the honeycombs of bees. Blood enters a lobule via the portal tracts (PT), located at the corners. Each PT contains a hepatic arteriole (supplying the liver with oxygenated blood), a portal venule (providing nutrient-rich blood from the intestines), and a bile ductule. The latter drains the bile produced in the lobule in the opposite direction from the blood flow in the PT. A fraction of the blood from the PT enters the vascular septa (VS), which are the vascular surfaces between neighbouring lobules, bordered at opposite edges by two PTs (Fig. 1). As such, the VS foresee the blood supply of the tissue between PTs by a number of microvessels branching off the PTs. Blood flows from the PTs and VS into the interconnected network of tortuous sinusoids, which are the hepatic-specific capillaries and are lined by a fenestrated endothelial cell layer. Blood solutes can flow through the fenestrations into the space of Disse, which is the space between the endothelial cells and the hepatocytes. Here, the metabolic exchange (e.g. oxygen or nutrient uptake) takes place with the neighbouring hepatocytes. The blood is further drained from the sinusoids into the central vein $(\mathrm{CV})$, and from there into the hepatic veins and vena cava inferior $[2,3]$. Due to the liver's microvascular organisation and its metabolic functions, liver lobules are characterized by metabolic zonation $[2,10]$. For example, the cell zone closest to the 
PTs will receive blood that is rich in nutrients and oxygen, while the zone close to the $\mathrm{CV}$ receives blood that is poorer in nutrients and oxygen. Although the liver lobule is the most frequently used functional liver unit, a number of other units has been proposed in the past, such as the hepatic acinus [11] (dotted line in Fig. 1b) and the primary lobule [7, 8]. Nevertheless, the actual real 3D structure of the liver microcirculation seems to be more complicated, as was illustrated in one of our previous papers [12], on the basis of a high resolution 3D micro-CT dataset of the human liver microcirculation, and liver lobules are not always perfect prisms. Additionally, variations are frequently observed in the number of PTs per lobule (polyhedral lobules with four, five or seven corners instead of six).

Until now, our knowledge of the liver microcirculation is mainly based on schematic concepts, and its hemodynamic behavior is still not fully understood. Especially in the case of abnormal liver conditions, the liver microcirculation plays a crucial role: when impaired or damaged (for instance due to disease), this can result in a loss of liver function due to the tight coupling between liver microperfusion and its metabolic function. Consequently, it is necessary to gain more insight into liver microvascular hemodynamics to also have a deeper understanding of function. Previously, numerical models have been used successfully to better understand the microcirculation. These models (or modelling techniques) might be helpful to understand microhemodynamics in a healthy liver as well as in liver pathology. Knowing that the microcirculation operates within relatively narrow ranges of hemodynamic conditions, microcirculation alterations might lead to biomechanical stressors (high shear stresses, high wall stresses). As such, these numerical models are applicable to investigate transplant-related phenomena (for which the preservation of the hepatic microcirculation is crucial for the viability of the liver graft $[13,14])$, 
pathology-related microvascular alterations (e.g. in the case of fibrosis, cirrhosis $[15$, 16], hepatocellular carcinoma [17], steatosis [18], portal hypertension [19, 20]), as well as surgery-induced effects (e.g. small-for-size liver syndrome [21-23] after partial hepatectomy). For example, cirrhosis is known to alter and deteriorate the liver's microvessel structure leading to higher vascular resistances, which could be modelled by changing structure-related parameters such as the permeability. Next to these applications, reduced order versions of these models may also be implemented in larger scale models (e.g. [1]) to capture the total liver hemodynamics, incorporating the macro- as well as the meso- and microcirculation and the interaction of the liver with other organs in whole system models.

However, only a few models on the topic of liver microhemodynamics have been published, which might be due to the complexity of the liver microcirculation and the difficulties associated with the acquisition of the required anatomical and functional data at sufficient resolution. A few studies focused on modelling the lobule hemodynamics based on porous medium approaches, in which the sinusoids are represented as pores imbedded in a parenchymal matrix. One such, Ricken et al. [24] developed a 2D biphasic model of the blood flow in a longitudinal cross-section of the liver lobules using a permeability that is isotropic over the cross-section. Bonfiglio et al. [25] studied the flow patterns in a 2D hexagonal cross-section in a porous lobule model, assuming both isotropic permeability as well as 2D anisotropic permeability (radial versus circumferential). Both of these models neglected the existence of VS [24, 25], leading to predictions of poor perfusion of the regions midway between neighbouring PTs in the paper of Bonfiglio et al. [25]. Given the need to perfuse every hepatocyte well, this is unlikely to occur in vivo. Consequently, Siggers et al. [26] used a 2D symmetric triangular porous medium model (representing one sixth of a hexagonal 
cross-section of a lobule), which they solved analytically to investigate the effect of VS. The PT inflows, VS inflows and central outflows were approximated as point sources, line sources and point sinks, respectively.

Although the porous medium models described above are useful to capture the overall hemodynamics, they are based on a number of key assumptions. The flow is assumed to be planar (2D), while it is known that the real liver microcirculation has a complex 3D structure [12]. In all but one section of the paper by Bonfiglio et al. [25], the permeability is assumed to be isotropic. Furthermore, VS were either neglected [24, 25] or modelled as a line source, implying a constant inflow into the sinusoids along the length of the VS [26]. However, in reality, the inflow from the VS is likely to be smaller further away from the PTs (due to a lower pressure within the VS there). Since these assumptions were not validated before, 3D models may be helpful to clarify this. Rani et al. [27] previously developed a finite volume blood flow model in a 3D geometry incorporating a terminal hepatic arteriole, portal venule and $\mathrm{CV}$, as well as two fenestrated sinusoids using non-Newtonian blood properties. Though the modelling approach was sophisticated, an idealized 3D geometry was used to locally represent the blood flow from the portal tract passing through two sinusoids into the central vein. More complex tortuous and interconnected sinusoids as well as the VS were not taken into account here, while these are relevant for the hemodynamics at this level. In recent work, we developed a 3D numerical model of the liver terminal circulation based on high resolution micro-CT data [12]. We demonstrated that the three-dimensional permeability tensor of a sinusoidal network displays significant anisotropy, which is in contrast with the isotropic permeability conditions assumed in the $2 \mathrm{D}$ porous models mentioned above [24-26]. Working in a cylindrical coordinate system with the $z$-axis along the central vein of a lobule (Fig. 2), we found the permeability tensor to be 
approximately diagonal with an axial component roughly two times that of the radial and azimuthal components, which were themselves approximately equal.

Combining the strengths of the previous models and extending them could lead to a more accurate model and a better understanding of the hepatic microhemodynamics. Therefore, the aim of this study was to develop a 3D porous medium model of the perfusion of a liver lobule, accounting for anisotropy in the permeability of the sinusoidal network and incorporating the VS as a separate volume zone within the model. In addition, we performed a parameter sensitivity analysis of the newly proposed model. 


\section{Materials and methods}

\subsection{Simulation geometry and mesh generation}

A geometry, representing a liver lobule with VS, was studied using a cylindrical coordinate system $(r, \theta, z)$ with its $z$-axis along the $\mathrm{CV}$ and its origin at the centre of the simulation geometry (Fig. 2a).

The lobule geometry (Fig. 2a) was assumed to be a hexagonal prism with crosssection defined by a circumscribing cylinder of diameter $1 \mathrm{~mm}[2,28]$, resulting in a hexagonal edge length of $0.5 \mathrm{~mm}$. Lobules are reported to have lengths of up to several millimetres [28], and in this study we used a length of $1 \mathrm{~mm}$ as a representative value. Reported measurements of the diameter of the $\mathrm{CV}$ and portal venule (corresponding to the major PT inflow) vary substantially in the literature [2, 9, 29-31], and in our model we chose representative values. As such, the $\mathrm{CV}$ was modelled as a cylinder of diameter $60 \mu \mathrm{m}$ parallel to the $z$-axis, and the PTs along the six parallel edges as cylinders, each of diameter $40 \mu \mathrm{m}$. VS zones were defined as the outermost $10 \mu \mathrm{m}$ depth on the six faces of the prism that delineate the borders with the neighbouring lobules (Fig. 2a).

The simulation geometry was meshed with Gambit ${ }^{\mathrm{TM}}$ (Ansys, Canonsburg, PA, USA). A mesh sensitivity study was performed by creating four meshes using the Cooper scheme with the linked surface meshes of the top and bottom plane of the geometry as sources, and applying an interval size of $24,12,6$ and $5 \mu \mathrm{m}$ resulting in $4.6 \cdot 10^{4}, 3.8 \cdot 10^{5}, 3.1 \cdot 10^{6}$ and $5.1 \cdot 10^{6}$ hexahedral volume elements, respectively. The mesh sensitivity analysis showed approximately constant results for models having $3.1 \cdot 10^{6}$ or more elements. Consequently, the mesh with a $6 \mu \mathrm{m}$ interval size corresponding to $3.1 \cdot 10^{6}$ elements was used. 


\subsection{Cell zone conditions and fluid properties}

The volume of the lobule, representing the sinusoids, was characterized as a porous medium, with fluid flow given by Darcy's law:

$$
\vec{v}_{\text {porous }}=-\frac{1}{\mu} K \nabla p
$$

where $p[\mathrm{~Pa}]$ is the sinusoidal blood pressure, $\mu[\mathrm{Pa} \cdot \mathrm{s}]$ the dynamic viscosity of blood, $K$ $\left[\mathrm{m}^{2}\right]$ the second order permeability tensor and $\vec{v}_{\text {porous }}[\mathrm{m} / \mathrm{s}]$ the Darcy velocity) [32]. The permeability tensor $K$ (consisting of permeability coefficients $k\left[\mathrm{~m}^{2}\right]$; eq. 2 ) is represented by a diagonal matrix in the cylindrical coordinate system $(r, \theta, z)$ :

$$
K=\left[\begin{array}{lll}
k_{r r} & k_{r \theta} & k_{r z} \\
k_{\theta r} & k_{\theta \theta} & k_{\theta z} \\
k_{z r} & k_{z \theta} & k_{z z}
\end{array}\right]=\left[\begin{array}{ccc}
k_{r r} & 0 & 0 \\
0 & k_{\theta \theta} & 0 \\
0 & 0 & k_{z z}
\end{array}\right] .
$$

In this study, two different permeability tensors were used: an isotropic and an anisotropic tensor. The anisotropic tensor was defined by the permeability coefficients found in [12], giving $k_{z z}=3.64 \cdot 10^{-14} \mathrm{~m}^{2}, k_{r r}=1.56 \cdot 10^{-14} \mathrm{~m}^{2}$ and $k_{\theta \theta}=1.75 \cdot 10^{-14} \mathrm{~m}^{2}$. Isotropic permeability conditions were defined by assuming that the isotropic permeability equals the anisotropic radial permeability coefficient, $k_{r r}=k_{\theta \theta}=k_{z z}=$ 1.56. $10^{-14} \mathrm{~m}^{2}$. This choice was based on the observation that the radial permeability coefficient has the biggest impact on the resulting volume flow rate at the lobule outflow (CV).

The porosity of the lobule, $\varepsilon$, equals the fraction of the volume occupied by the blood $\left(\mathrm{V}_{\text {sinusoids }}\right.$ ) divided by the total volume $\mathrm{V}_{\text {total }}$ (eq. 3 ), and we fixed its value at 0.143 , as found in [12]. It also equals the ratio of the magnitude of the Darcy velocity (the volume-averaged flux per unit area; $\vec{v}_{\text {porous }}[\mathrm{m} / \mathrm{s}]$ ) to the magnitude of the crosssectionally averaged physical velocity in the sinusoids $\left(\vec{v}_{p h \text { ysica }}[\mathrm{m} / \mathrm{s}]\right)$ : 


$$
\varepsilon=\frac{V_{\text {sinusoids }}}{V_{\text {total }}}=\frac{\left\|\vec{v}_{\text {porous }}\right\|}{\left\|\vec{v}_{\text {physical }}\right\|} .
$$

In the following, all reported velocities are Darcy velocities and we will denote $\vec{v}_{\text {porous }}$ simply by $\vec{v}$. In order to estimate the typical physical velocities within the sinusoids, the Darcy velocity should thus be divided by the porosity $\varepsilon$ (eq. 3).

For the model without VS, the VS zones were defined using the same permeability coefficients as for the lobule zone of the sinusoids. In the case of models with VS, VS were treated as porous media with a higher circumferential permeability due to the circumferential orientation of the vessels within the VS. As such, we used in the VS the lobular value of $k_{\theta \theta}$ multiplied by a factor of either 10 or 100 , but keeping the values of the radial and longitudinal coefficients, $k_{r r}$ and $k_{z z}$, the same as their values in the sinusoids. Note that, ideally, we would have altered the component of the permeability tensor in the exact direction of the VS (rather than $k_{\theta \theta}$ in the circumferential direction, which is approximately in the same direction), but in this paper we considered this slightly simpler case.

In total, six cases were simulated to investigate the influence of including VS and the usage of (an)isotropy. We denote these models as M0I, M0A, M10I, M10A, M100I, M100A, where ' 0 ' refers to no VS, ' 10 ' to VS with $k_{\theta \theta} 10$ times that in the lobule interior and ' 100 ' to VS with $k_{\theta \theta} 100$ times that in the lobule interior, and 'I' refers to isotropic permeability and 'A' to anisotropic permeability (Table 1). Blood was assumed to be an incompressible Newtonian fluid with a dynamic viscosity of 3.5 $\mathrm{mPa} \cdot \mathrm{s}[33]$. 


\subsection{Boundary conditions and computational fluid dynamics}

For all models, the top and bottom hexagonal planes were characterized by a translational periodic boundary condition (Fig. 2b), and we assumed $1 \mathrm{mmHg} / \mathrm{mm}$ to be the $z$-component of the pressure gradient. The pressure was defined to drop from 6.18 $\mathrm{mmHg}$ to $5.18 \mathrm{mmHg}$ along the PT inflow and from $5.23 \mathrm{mmHg}$ to $4.23 \mathrm{mmHg}$ along the $\mathrm{CV}$ outflow (corresponding to mean pressures of $5.68 \mathrm{mmHg}$ at the PT and 4.73 $\mathrm{mmHg}$ at the $\mathrm{CV}$, as found in [30]). At the planes of interface between the lobule and VS, continuity of pressure and flux was applied. Since we assumed a repeating honeycomb pattern (Fig. 1b), we used symmetry boundary conditions at the surfaces delineating the lobule borders.

The steady computational fluid dynamic models were solved using Fluent ${ }^{\mathrm{TM}}$ (Ansys, Canonsburg, PA, USA). Tecplot ${ }^{\mathrm{TM}}$ (Tecplot Inc., Bellevue, WA) was used to process, calculate and visualize the hemodynamic parameters of interest.

\subsection{Parameter sensitivity study}

Since some assumptions were made concerning the boundary conditions and simulation geometry, with some degree of variability in values reported in literature, we performed a parameter sensitivity analysis of the model. The M100A model was used as the baseline model to perform parameter variations of the pressure boundary conditions as well as geometry-related parameters (see also Table 1).

As such, the longitudinal pressure gradient was changed from the default value of $1 \mathrm{mmHg} / \mathrm{mm}$ to $0 \mathrm{mmHg} / \mathrm{mm}$ (M100A_1), $0.5 \mathrm{mmHg} / \mathrm{mm}$ (M100A_2) and 2 mmHg/mm (M100A_3). The pressure drop between the PTs and CV was changed by increasing the default PT pressure, defined to drop from $6.18 \mathrm{mmHg}$ to $5.18 \mathrm{mmHg}$ along the PT inflow, by $0.5 \mathrm{mmHg}$ (M100A_4) and 1 mmHg (M100A_5). 
The thickness of the VS was changed to $5 \mu \mathrm{m}$ (M100A_6) instead of $10 \mu \mathrm{m}$, and the PT (M100A_7) and CV diameters (M100A_8) were increased by 50\% of their original value. Additionally, the effect of the lobule circumferential diameter was investigated by scaling down the lobule. Note that if we would consider a scale model in which we change all length scales (lobule circumferential diameter, and the radii of the CV and PTs) in the same way, the flux would remain unchanged, since the velocity scale is inversely proportional to the length scale. Consequently, we considered a lobule circumferential diameter of $0.5 \mathrm{~mm}$ instead of $1 \mathrm{~mm}$, but kept the radii of the PT and CV the same (M100A_9). A summary of all simulated cases and the corresponding parameters is shown in Table 1. 


\section{Results}

We first compare the hemodynamics of the models with and without VS (Section 3.1), followed by a comparison of the isotropic and anisotropic permeability cases (Section 3.2). The results of the parameter sensitivity analysis are reported in section 3.3.

\subsection{Comparison of the models with and without vascular septa}

Since the impact of VS on the results for the isotropic and anisotropic models are qualitatively similar (Figs. 3-7), we only describe here the results for the anisotropic models in detail.

\subsubsection{Flow in the hexagonal cross-sectional plane in the middle of the lobule $(z=0)$}

In model M0A, over the hexagonal cross-section $z=0$ the pressure drops from $5.68 \mathrm{mmHg}$ at the PTs to $5.40 \mathrm{mmHg}$ at the points midway between neighbouring PTs to $4.73 \mathrm{mmHg}$ at the $\mathrm{CV}$ (Fig. 3a, 4b). The projection of the velocity into the crosssection, $\left\|\vec{v}_{r \theta}\right\|$, has a relatively high magnitude at the entry from the PTs (Fig. 3b). From there the blood flows to the $\mathrm{CV}$, either directly, or first towards the regions midway between neighbouring PTs and then towards the CV. Along each pathway, the crosssectional velocity decreases away from the PT, reaching a streamline-specific minimum (with the lowest value being a zero velocity, which is attained at the stagnation points at the outer lobule borders midway between neighbouring PTs; Fig. 3c) before rising to its highest value at the CV outlet (Fig. 3d).

As can be seen in Figs. 4d, f, the model demonstrates different hemodynamics when VS are present. The pressure drop from the PT to the VS is smaller in M10A and M100A than in the case without VS (Fig. 3a), and the pressure drop from the centre of 
the VS to the CV is larger. There are two flow pathways: those pathways on which fluid enters the sinusoids directly from the PTs; and those on which fluid travels through the VS before entering the sinusoids. Within the VS, the flow is predominantly circumferential, and the cross-sectional velocities at the PT-VS interface are high. At the CV outflow the velocities are approximately spatially uniform (Fig. 3d, Fig. 4 d, f). On leaving the PTs, the flow pathways that go directly from the PT into the sinusoids have lower velocities than those that first enter the VS and also than those leaving the PTs in model M0A (see Fig. 3b). The streamlines in models M10A and M100A are predominantly radial, and the regions midway between neighbouring PTs are better perfused, leading to a more uniform distribution of blood throughout the tissue than in M0A (Fig. 4). This effect is more pronounced for higher circumferential permeability in the VS, i.e. more in M100A than in M10A. Moreover, as shown in Table 2, the volumetric flow rate at the outflow of the lobule $\left(\mathrm{Q}_{\text {lobule }}\right)$ is higher when including VS $\left(6.58 \cdot 10^{-5} \mathrm{ml} / \mathrm{min}\right.$ and $6.78 \cdot 10^{-5} \mathrm{ml} / \mathrm{min}$ for M10A and M100A, respectively) than when neglecting $\operatorname{VS}\left(6.02 \cdot 10^{-5} \mathrm{ml} / \mathrm{min}\right.$ for $\left.\mathrm{MOA}\right)$.

\subsubsection{Flow in the longitudinal cross-section through opposite PTs $\left(\theta=0^{\circ}, 180^{\circ}\right)$}

Fig $5 \mathrm{~b}$ shows the pressure and streamlines in M0A in a longitudinal plane of cross-section containing the axis of the lobule and two PTs. The flow follows a diagonally downward pathway, due to the combined effects of the imposed $z$ component of the pressure gradient and of the radial pressure gradient. The velocity is high at the inflow (Fig. 3e), lower midway between the PT and the CV, and then higher near the CV outflow. 
Similar flow patterns are found in M10A and M100A (Figs. 5d, f); however, these models have lower velocities at the PT inflow (Fig 3e), and higher velocities at the CV outflow.

3.1.3 Flow in the longitudinal cross-section through the centrelines of opposite VSs ( $\theta$ $\left.= \pm 90^{\circ}\right)$

Fig. $6 \mathrm{~b}$ shows the pressure in M0A in a longitudinal plane of cross-section containing the axis of the lobule and passing through the midpoints of two VSs. The pressure decreases from the outer boundary to the CV outflow. Flow velocities in this plane are generally smaller than the velocities in the plane passing through the PTs, especially near the peripheral boundaries, where there is a stagnation line with a zero velocity midway between two PTs along the outer lobule borders.

Comparing the models with and without VS, we find that the pressures in M10A and M100A are generally higher than those in M0A, see Fig. 6b, d, f. The planar velocity magnitudes at the VS-lobule interfaces are $1.40 \cdot 10^{-6} \mathrm{~m} / \mathrm{s}$ and $1.43 \cdot 10^{-6}$ for M10A and M100A, respectively (see Fig. 3f). However, velocities at the CV outflow are higher.

In the end, the models without VS show low magnitudes of velocity and thus poor perfusion of the zones lying between neighbouring PTs (Figs. 4a-b, 7a-b). In contrast, the models with VS predict a better perfusion of these zones and a more uniform velocity distribution (Figs. 4c-f, 7a-b). Figs. 7c-d show, respectively, the inplane and the total (3D) magnitudes of the velocity as a function of the angle on a circle around the $\mathrm{CV}(r=0.3 \mathrm{~mm})$, in order to investigate the homogeneity of the perfusion. 
The flow is more homogeneous when VS are included, with the amplitude of the velocity magnitude fluctuations clearly being smaller when including VS.

\subsection{Comparison of isotropic versus anisotropic permeability}

In this section we discuss isotropic versus anisotropic conditions, focusing on models M100I and M100A.

In the hexagonal cross-section $z=0$, the pressure contours and directions of the flow trajectories are similar in the isotropic and the anisotropic models (Fig. 4e-f). The in-plane magnitudes of the velocity as well as the total flow rate through the lobule are almost equal (slightly higher in the anisotropic cases): $6.77 \cdot 10^{-5} \mathrm{ml} / \mathrm{min}$ in M100I and 6.79. $10^{-6} \mathrm{ml} / \mathrm{min}$ in M100A (Table 2).

Figs. 5e-f and 6e-f show that the flow is similar for the corresponding cases of isotropic and anisotropic permeability, although it is closer to vertical in the anisotropic case. As such, anisotropic permeability leads to a larger longitudinal z-component of the velocity, due to the larger longitudinal permeability in the $z$-direction. Similar effects are observed in the other models: M0I, M0A, M10I and M10A.

Fig. 7c shows that the planar velocity along a circle around the CV in the hexagonal cross-section is approximately equal for both the anisotropic and isotropic cases, with isotropic conditions resulting in only a slightly higher variation of the predicted velocity. However, the spatially averaged magnitude of the total (3D) velocity is significantly higher and also shows a smaller spatial variance in the anisotropic case, owing to the higher $z$-component of the velocity (Fig. 7d). 


\subsection{Parameter sensitivity study}

Boundary conditions as well as geometrical features were varied to investigate the sensitivity of the lobule fluid-dynamics to the choice of such parameters.

\subsubsection{Impact of changes in imposed boundary conditions}

Comparing Fig. 5f with Fig. 8 illustrates how changes of the pressure gradient in the $z$-direction affect the solution. Increasing the longitudinal pressure gradient leads, as expected, to a larger $z$-component of the velocity. In contrast, the lobule and total liver outflow and resistance do not change in comparison with the baseline model M100A (Table 1 and 2). Obviously, the radial and circumferential components of the velocity do not depend on the longitudinal pressure gradient, whereas the $z$-component of the velocity is directly proportional to $\partial p / \partial z$. As the total flux through the lobule only depends on the radial and circumferential velocity components, it is also independent of $\partial p / \partial z$

Secondly, the PT-CV pressure drop was increased by increasing the PT pressure by $0.5 \mathrm{mmHg}$ and $1 \mathrm{mmHg}$ (Fig. 9). This resulted in higher velocities and lobule flows $\left(1.03 \cdot 10^{-4} \mathrm{ml} / \mathrm{min}\right.$ and $1.39 \cdot 10^{-4} \mathrm{ml} / \mathrm{min}$ for a $0.5 \mathrm{mmHg}$ and $1 \mathrm{mmHg}$ increase of the PT pressure, respectively), because the flow is given by dividing the pressure difference by the resistance (see also the Appendix for details). The lobule resistance remained equal, since the simulation geometry did not change (Table 1 and 2). Lobule and total liver flow increases were linearly proportional to the increase of the PT-CV pressure drop (Table 2). Note that this dependence of the results on the PT-CV pressure difference is predictable, owing to the linearity of the governing equations: the radial and circumferential components of the velocity (and consequently also the flux through the lobule) are directly proportional to the PT-CV pressure drop, while such difference 
does not affect the $z$-component of the velocity. Doubling the PT-CV pressure drop, for instance, thus implies doubling the flux through the lobule.

\subsubsection{Impact of geometry -related parameter variations}

Reducing the VS thickness to $5 \mu \mathrm{m}$ (instead of $10 \mu \mathrm{m}$ ) results in a decrease of the fraction of the PT inflow that drains into the VS (Fig. 10). This led to an increase in the lobule resistance $\left(1.46 \cdot 10^{4} \mathrm{mmHg} \cdot \mathrm{min} / \mathrm{ml}\right)$ and a decrease of the lobule flow $\left(6.51 \cdot 10^{-5} \mathrm{ml} / \mathrm{min}\right)$. As such, decreasing the VS thickness by $50 \%$ resulted in a lobule and total liver outflow decrease of $4.0 \%$ (Table 2).

Increasing the PT diameter from 40 to $60 \mu \mathrm{m}$ results in a decrease of the lobule resistance $\left(1.35 \cdot 10^{4} \mathrm{mmHg} \cdot \mathrm{min} / \mathrm{ml}\right)$. Consequently, the lobule outflow increased $\left(7.01 \cdot 10^{-5} \mathrm{ml} / \mathrm{min}\right)$. Pressures and velocities changed accordingly, as illustrated in Fig. 10 (lower PT inflow velocity but a larger PT inflow front, higher CV outflow velocity). A $50 \%$ increase of the PT diameter thus resulted in a $3.4 \%$ increase of the lobule and total liver outflow (Table 2).

When increasing the CV diameter to $90 \mu \mathrm{m}$ (instead of $60 \mu \mathrm{m}$; Fig. 10), the lobule outflow increases $\left(7.80 \cdot 10^{-5} \mathrm{ml} / \mathrm{min}\right)$ while the lobule resistance decreases due to a larger $\mathrm{CV}$ outflow front $\left(1.22 \cdot 10^{4} \mathrm{mmHg} \cdot \mathrm{min} / \mathrm{ml}\right)$. As such, a $50 \% \mathrm{CV}$ diameter increase results in a $15.1 \%$ increase of the lobule and total liver outflow (Table 2).

Furthermore, when reducing the lobule circumferential diameter to $0.5 \mathrm{~mm}$ (Fig. $10)$, the result is a significant increase in the lobule flow $\left(9.74 \cdot 10^{-5} \mathrm{ml} / \mathrm{min}\right)$, implying that the lobule resistance to flow is smaller $\left(9.75 \cdot 10^{3} \mathrm{mmHg} \cdot \mathrm{min} / \mathrm{ml}\right)$. In addition, a smaller lobule results in a larger number of lobules in a liver of a given size, and leads in this case to a total estimated liver flow of $900 \mathrm{ml} / \mathrm{min}$. Consequently, a $50 \%$ decrease 
of the lobule circumferential diameter leads to a $43.7 \%$ increase of the lobule outflow and a $474.8 \%$ increase of the total liver outflow (Table 2).

In summary, amongst the model parameters studied, the lobule circumferential diameter is the most sensitive parameter when looking at the total liver outflow, followed by the pressure drop between the PTs and the CV. 


\section{Discussion}

In this study, a 3D computational fluid dynamics model of a liver lobule was developed to study the hepatic microcirculation in order to investigate the effect of vascular septa and anisotropic permeability properties. Additionally, a parameter sensitivity analysis was performed to study the influence of the choice of geometric and physic parameters on the lobule fluid-dynamics.

The models with VS clearly show better perfusion of the zones lying between neighbouring PTs compared to the models without VS, and the flow patterns are more homogeneous when VS are included. The physiological needs of the hepatocytes, and, in particular the requirement of a sufficient supply of oxygen and nutrients for every cell, make the models that include VS seem more physiologically plausible than those without. This is particularly the case for the cells midway between neighbouring PTs whose supply of oxygen is dramatically increased by the existence of VS.

Models with anisotropic permeability show a more spatially homogeneous magnitude of the velocity field compared to those having isotropy (Fig. 7). Again, this is especially relevant for the relatively poorly perfused zone midway between neighbouring PTs. However, one has to be cautious with this interpretation, since the influence of anisotropy depends on the applied boundary conditions, in particular on the longitudinal pressure gradient. To the authors' knowledge, there are no data available in literature on the longitudinal pressure gradient over a lobule (defined in our model as 1 $\mathrm{mmHg}$ per mm; see section 2.2). Therefore, we performed a parameter study in which variations of the longitudinal pressure gradient showed as expected differences in the zcomponents of the velocities, but the lobule and total liver flow did not change (Table 1 and 2). 
In addition, the sensitivity of other parameters was analysed (Table 1 and 2), showing that the total liver outflow was most sensitive to changes in the lobule crosssectional size (determined by the lobule circumferential diameter) and the PT-CV pressure drop. In contrast, the total liver outflow was not or only slightly sensitive to some other parameters (such as the longitudinal pressure gradient in the z-direction and the PT diameter).

Estimations of the flow rate and resistance of both a single lobule and the liver as a whole for M100A resulted in $Q_{\text {lobule }}=6.79 \cdot 10^{-5} \mathrm{ml} / \mathrm{min}, Q_{\text {liver }}=157 \mathrm{ml} / \mathrm{min}, R_{\text {lobule }}$ $=1.40 \cdot 10^{4} \mathrm{mmHg} \cdot \mathrm{min} / \mathrm{ml}$ and $R_{\text {liver }}=6.06 \cdot 10^{-3} \mathrm{mmHg} \cdot \mathrm{min} / \mathrm{ml}$, respectively $($ Table 2). However, physiological values of $Q_{\text {liver }}$ are around $1500 \mathrm{ml} / \mathrm{min}$, which our baseline model underestimates. This is due to the assumptions we made in our modelling, which is not surprising since there are significant variations of certain parameters reported in the literature (PT and CV pressures and diameters, lobule dimensions, liver weight and flow rate etc.). We anticipate that, as better estimates of these parameters become available, the model will become more realistic and can be tuned based on these parameters so that it effectively leads to realistic whole-liver flow characteristics. For example, with a lobule diameter of $0.5 \mathrm{~mm}$ and a pressure in the PT of $0.5 \mathrm{mmHg}$ or 1 $\mathrm{mmHg}$ above the default values, the flow rate through the whole liver is $1380 \mathrm{ml} / \mathrm{min}$ (M100A_10) or $1850 \mathrm{ml} / \mathrm{min}$ (M100A_11), respectively, and these values are within the physiological range (Table 2).

It is important to stress that we did not perform a direct validation of the proposed models, which would require measurements of pressures or flows at the liver microcirculation level, which is very challenging. However, our results correspond to values that have been described in the literature, and we expect the qualitative predictions of our model to be valid. The demonstrated differences in the flow patterns, 
especially in the presence of VS, are independent of the assumed sets of boundary conditions. As such, our models incorporating VS and anisotropic permeabilities are probably more accurate since they result in more homogeneous flow patterns compared to previous models.

When comparing the outcomes of our models to literature, the highest magnitudes of velocity were found near the inflow from the PTs and near the CV, which agrees with the findings of Bonfiglio et al. and Ricken et al. [24, 25]. Cohen et al. [34] also stated that the velocity near the CV is higher, resulting in a region of hepatocytes near the $\mathrm{CV}$ that have a much higher flux of blood passing them, as compared with hepatocytes that are located nearer the periphery. The flow patterns in the longitudinal sections through the PTs shown in Fig. 5 are also similar to those reported earlier by Ricken et al. [24]. However, in their study, the streamlines are closer to those we obtained in our isotropic models, and they are orientated further from the axis than the streamlines we obtained in our anisotropic models. This seems to be a logical consequence of the fact that Ricken et al. [24] used a permeability that is isotropic over the longitudinal cross-section in his $2 \mathrm{D}$ model. The flow patterns in the hexagonal cross-sectional plane of the models without VS (shown in Fig. 4 a, b), are also qualitatively similar to those obtained by Bonfiglio et al. [25]. Furthermore, the results obtained by Siggers et al. [26] in their dimensionless 2D model including VS were comparable with our models including VS (projection into the hexagonal crosssectional plane). Note that the 3D lobule model obtained in this study could also be interpreted and solved as a combination of a $2 \mathrm{D}$ porous model $[25,26]$ with a superimposed pressure gradient in the $z$ direction and the corresponding $z$-component of the velocity, since our model uses a prescribed longitudinal pressure drop. Comparing our model with the 3D model of Rani et al. [27], some major differences exist regarding 
the modelling approach. Rani et al. [27] based the fluid domain of their model on the 3D structure of a liver acinus, while in this study we used the classic schematic hexagonal lobule to represent the functional unit of the liver microcirculation (Fig. 1). Which model is most appropriate to represent the functional unit of the liver (e.g. the classic lobule, primary lobule, liver acinus, choleohepaton etc.) is still a matter of debate $[2,5,9,29,35,36]$. Furthermore, the model of Rani et al. [27] included a terminal HA, PV and CV as well as two sinusoids having a $23.5 \mu \mathrm{m}$ radius and fenestrations. These sinusoidal diameter values seem to be high, since sinusoidal diameters are typically reported as being in the order of magnitude of $10 \mu \mathrm{m}$ (which we also observed in a previous study [12]). Larger sinusoids will result in lower vascular resistances and higher permeability compared to our model geometry. Another difference is that Rani et al. [27] used non-Newtonian fluid properties, while we modelled blood as an incompressible Newtonian fluid. Non-Newtonian effects (such as the Fahraeus-Lindqvist effect [37] and the shear thinning effect [27]) are important on the scale of the microscopic sinusoids. Nevertheless, Bonfiglio et al. [25] reported pressure differences of less than $4 \%$ when comparing the results of a Newtonian model with those of a shear thinning model. This seems to suggest that non-Newtonian effects are of minor importance for our models. As for the boundary conditions, Rani et al. [27] applied a pressure drop from $95 \mathrm{mmHg}$ to $20 \mathrm{mmHg}$ along the terminal $\mathrm{HA}$ and from $25 \mathrm{mmHg}$ to $15 \mathrm{mmHg}$ along the terminal PV, which seems quite high (a PV pressure of $15-25 \mathrm{mmHg}$ is considered indicating portal hypertension). Thus, combining higher pressure(s) (drops) with lower vascular resistances (due to higher sinusoidal diameters) leads to significant differences in the order of magnitude of the predicted velocities with our data. Furthermore, the model of Rani et al. [27] explicitly accounts neither for the effect of more than two tortuous sinusoids, nor for the presence of VS. 


\section{Conclusion}

In this study, we developed a 3D mathematical model of a liver lobule, using a porous medium approach, incorporating the effect of vascular septa and anisotropic permeability. The inclusion of vascular septa leads to better perfusion of the zones lying between portal tracts as well as a more spatially homogeneous perfusion of the lobule. The inclusion of anisotropic permeability results in clearly different flow patterns, with streamlines that are oriented closer to the lobule axes. A parameter study revealed that the lobule and total liver flow is most sensitive to variations of the lobule circumferential diameter and the radial pressure drop. In conclusion, the findings suggest that especially the presence of vascular septa results in a more physiologically realistic model of the hepatic microcirculation, and that the model can be tuned to correspond with physiological flows and pressures. Future work should aim at direct validation of the model and to explore applications for a better understanding of liver pathophysiology as well as improved diagnosis and treatment of liver disease.

\section{Acknowledgements}

This research was supported by the Agency for Innovation by Science and Technology in Flanders (IWT), Belgium. Diethard Monbaliu holds a chair of the Centrale Afdeling voor Fractionering (CAF), Vilvoorde, Belgium.

\section{Nomenclature}

$=2$-dimensional

3D

$=3$-dimensional

K

$=$ permeability tensor 


$\begin{array}{ll}p[\mathrm{~Pa}] & =\text { pressure } \\ Q\left[\mathrm{~m}^{3} / \mathrm{s}\right] & =\text { flow } \\ R[\mathrm{mmHg} \cdot \mathrm{min} / \mathrm{ml}] & =\text { vascular resistance } \\ V\left[\mathrm{~m}^{3}\right] & =\text { volume } \\ k\left[\mathrm{~m}^{2}\right] & =\text { permeability coefficient } \\ \vec{v} & =\text { velocity vector } \\ \varepsilon & =\text { porosity } \\ \mu[\mathrm{Pa} \cdot \mathrm{s}] & =\text { dynamic viscosity } \\ \rho\left[\mathrm{kg} / \mathrm{m}^{3}\right] & =\text { density }\end{array}$




\section{Appendix: estimation of the lobe-specific and total liver flow and resistance}

Based on the simulation results, the flow rate in a single lobule, $Q_{\text {lobule }}\left[\mathrm{m}^{3} / \mathrm{s}\right]$, can be estimated by multiplying the surface area of the $\mathrm{CV}$ by a typical normal velocity component of the flux entering the $\mathrm{CV}$

$$
Q_{\circ}=\vec{b}\left\|\vec{v}_{C}\right\|_{i} A_{C}=\| \vec{v}_{C} \mid 2 \pi r_{C} \dot{v}_{l} \text { o, }
$$

where $\vec{v}_{C V}[\mathrm{~m} / \mathrm{s}]$ is the radial velocity at the CV outflow, $A_{C V}\left[\mathrm{~m}^{2}\right]$ is the surface area of the CV lumen, $r_{c v}\left[30 \cdot 10^{-6} \mathrm{~m}\right]$ is the $\mathrm{CV}$ radius, and $l_{\text {lobule }}\left[10^{-3} \mathrm{~m}\right]$ the lobule length. The total liver flow, $Q_{\text {liver }}\left[\mathrm{m}^{3} / \mathrm{s}\right]$, is calculated by multiplying $Q_{\text {lobule }}$ by the number of lobules, $n$, which is estimated by dividing a typical volume of the liver by the volume of a lobule (hexagonal prism volume):

$$
n=\frac{V_{\text {liver }}}{V_{\text {lobule }}}=\frac{\begin{array}{l}
Q_{\text {ive }} \\
3 \cdot r_{\text {lobul }} \frac{m_{\text {liver }}}{\rho_{\text {liver }}} \\
\frac{\sqrt{3} r_{\text {lobule }}}{2} \cdot l_{\text {lobul }}
\end{array}}{\text {, }}
$$

where $V_{\text {liver }}\left[\mathrm{m}^{3}\right]$ is the liver volume, $V_{\text {lobule }}\left[\mathrm{m}^{3}\right]$ is the lobule volume, $m_{\text {liver }}[1.5 \mathrm{~kg}]$ is the mass of the liver [3], $\rho_{\text {liver }}\left[10^{3} \mathrm{~kg} / \mathrm{m}^{3}\right.$; equal to water] the liver density and $r_{\text {lobule }}$ the length of a side of the lobule hexagonal cross-section. The lobule-specific hydraulic resistance, $R_{\text {lobule }}\left[\mathrm{Pa} \cdot \mathrm{s} / \mathrm{m}^{3}\right]$, is estimated by dividing the pressure difference between the inflow and outflow (along the streamlines running from PT to CV) by the lobule-specific flow:

$$
R_{\text {lobule }}=\frac{\Delta P_{\text {lobule }}}{Q_{\text {lobule }} .}
$$

Since the lobules are arranged in parallel with respect to the blood flow, the total hydraulic resistance of the microvasculature of the liver is calculated as the lobule resistance divided by the estimated number of lobules $n$ : 


$$
R_{\text {liver }}=\frac{R_{\text {lobule }}}{n} \text {. }
$$




\section{References}

[1] Debbaut C, Monbaliu D, Casteleyn C, Cornillie P, Van Loo D, Masschaele B, Pirenne J, Simoens P, Van Hoorebeke L, and Segers P. 2011. From vascular corrosion cast to electrical analog model for the study of human liver hemodynamics and perfusion. IEEE Transactions on Biomedical Engineering. 58(1):25-35.

[2] Burt AD, Portmann BC, and Ferrell LD. 2007. MacSween's Pathology of the liver. Churchill Livingstone.

[3] E. N. Marieb KH. 2008. Anatomy \& physiology. 3rd edition. San Francisco: Pearson Education.

[4] Monbaliu D, Debbaut C, Hillewaert W, Brassil J, Laleman W, Sainz-Barriga M, Kravitz D, Pirenne J, and Segers P. 2012. Flow competition between hepatic arterial and portal venous flow during hypothermic machine perfusion preservation of porcine livers. International Journal of Artificial Organs. 35(2):119-131.

[5] Ekataksin W, and Wake K. 1991. Liver units in 3 dimensions.1. Organization of argyrophilic connective-tissue skeleton in porcine liver with particular reference to the compound hepatic lobule. American Journal of Anatomy. 191(2):113-153.

[6] Greenway CV, and Stark RD. 1971. Hepatic vascular bed. Physiol. Rev. 51(1):23-65.

[7] Matsumoto T, and Kawakami M. 1982. The unit-concept of hepatic parenchyma-a re-examination based on angioarchitectural studies. Acta Pathologica Japonica. 32(2):285-314. 
[8] Matsumoto T, Komori R, Magara T, Ui T, Kawakami M, Tokuda T, Takasaki S, Hayashi H, Jo K, Hano H, Fujino H, and Tanaka H. 1979. A study on the normal structure of the human liver, with special reference to its angioarchitecture. Jikeikai Med. 26:1-40.

[9] Teutsch HF, Schuerfeld D, and Groezinger E. 1999. Three-dimensional reconstruction of parenchymal units in the liver of the rat. Hepatology. 29(2):494-505.

[10] Zou Z, Ekataksin W, and Wake K. 1998. Zonal and regional differences identified from precision mapping of vitamin A-storing lipid droplets of the hepatic stellate cells in pig liver : A novel concept of addressing the intralobular area of heterogeneity. Hepatology. 27(4):1098-1108

[11] Rappaport AM, Borowy ZJ, Lougheed WM, and Lotto WN. 1954. Subdivision of hexagonal liver lobules into a structural and functional unit; role in hepatic physiology and pathology. Anat Rec. 119(1):11-33.

[12] Debbaut C, Vierendeels J, Casteleyn C, Cornillie P, Van Loo D, Simoens P, Van Hoorebeke L, Monbaliu D, and Segers P. 2012. Perfusion characteristics of the human hepatic microcirculation based on three-dimensional reconstructions and computational fluid dynamic analysis. J. Biomech. Eng. 134(1)

[13] Monbaliu D, and Brassil J. 2010. Machine perfusion of the liver: past, present and future. Current Opinion in Organ Transplantation. 15(2):160-166.

[14] Monbaliu D, Vekemans K, De Vos R, Brassil J, Heedfeld V, Qiang L, D'Hollander M, Roskams T, and Pirenne J. 2007. Hemodynamic, biochemical, and morphological characteristics during preservation of normal porcine livers by hypothermic machine perfusion. Transplantation Proceedings. 39(8):2652-2658. 
[15] Chen ML, Zeng QY, Huo JW, Yin XM, Li BP, and Liu JX. 2009. Assessment of the hepatic microvascular changes in liver cirrhosis by perfusion computed tomography. World Journal of Gastroenterology. 15(28):3532-3537.

[16] Vanheule E, Geerts AM, Van Huysse J, Schelfhout D, Praet M, Van Vlierberghe H, De Vos M, and Colle I. 2008. An intravital microscopic study of the hepatic microcirculation in cirrhotic mice models: relationship between fibrosis and angiogenesis. Int. J. Exp. Pathol. 89(6):419-432.

[17] Maksan SM, Paulo H, Ryschich E, Kuntz C, Gebhard MM, Klar E, and Schmidt J. 2003. In vivo assessment of angioarchitecture and microcirculation in experimental liver cancer - a new model in rats. Dig. Dis. Sci. 48(2):279-290.

[18] Rosenstengel S, Stoeppeler S, Bahde R, Spiegel HU, and Palmes D. 2011. Type of steatosis influences microcirculation and fibrogenesis in different rat strains. J. Invest. Surg. 24(6):273-282.

[19] Fondevila C, Hessheimer AJ, Taura P, Sanchez O, Calatayud D, de Riva N, Munoz J, Fuster J, Rimola A, and Garcia-Valdecasas JC. 2010. Portal hyperperfusion: mechanism of injury and stimulus for regeneration in porcine small-for-size transplantation. Liver Transplantation. 16(3):364-374.

[20] Yagi S, Lida T, Taniguchi K, Hori T, Hamada T, Fujii K, Mizuno S, and Uemoto S. 2005. Impact of portal venous pressure on regeneration and graft damage after living-donor liver transplantation. Liver Transplantation. 11(1):68-75.

[21] Glanemann M, Eipel C, Nussler AK, Vollmar B, and Neuhaus P. 2005. Hyperperfusion syndrome in small-for-size livers. European Surgical Research. $37(6): 335-341$. 
[22] Ikegami T, Shimada M, Imura S, Arakawa Y, Nii A, Morine Y, and Kanemura H. 2008. Current concept of small-for-size grafts in living donor liver transplantation. Surgery Today. 38(11):971-982.

[23] Tanaka K, and Ogura Y. 2004. "Small-for-size graft" and "small-for-size syndrome" in living donor liver transplantation. Yonsei Medical Journal. 45(6):10891094.

[24] Ricken T, Dahmen U, and Dirsch O. 2010. A biphasic model for sinusoidal liver perfusion remodeling after outflow obstruction. Biomechanics and Modeling in Mechanobiology. 9(4):435-450.

[25] Bonfiglio A, Leungchavaphongse K, Repetto R, and Siggers JH. 2010. Mathematical modeling of the circulation in the liver lobule. J. Biomech. Eng. 132(11):111011.

[26] Siggers JH, Bonfiglio A, Leungchavaphongse K, and Repetto R. 2010. Mathematical modeling of blood circulation in the liver. Paper presented at: 6th World Congress of Biomechanics (WCB 2010) in conjunction with 14th International Conference on Biomedical Engineering (ICBME) and 5th Asia Pacific Conference on Biomechanics (APBiomech): IFMBE Proceedings. 1-6 August 2010 Singapore.

[27] Rani HP, Sheu TWH, Chang TM, and Liang PC. 2006. Numerical investigation of non-Newtonian microcirculatory blood flow in hepatic lobule. Journal of Biomechanics. 39(3):551-563.

[28] Mareels G. 2008. Experimental and numerical modeling of flow and mass transport in a bio-artificial liver [PhD dissertation], [Gent (Belgium)]: Ghent University. 
[29] Teutsch HF. 2005. The modular microarchitecture of human liver. Hepatology. 42(2):317-325.

[30] Maass-Moreno R, and Rothe CF. 1997. Distribution of pressure gradients along hepatic vasculature. American Journal of Physiology-Heart and Circulatory Physiology. 272(6):H2826-H2832.

[31] Nakata K, Leong GF, and Brauer RW. 1960. Direct measurement of blood pressures in minute vessel of the liver. American Journal of Physiology. 199(6):11811188.

[32] Liakopoulos AC. 1965. Darcy's coefficient of permeability as symmetric tensor of second rank. International Association of Scientific Hydrology. 10(3):41-48.

[33] van der Plaats A, 't Hart NA, Verkerke GJ, Leuvenink HGD, Verdonck P, Ploeg RJ, and Rakhorst G. 2004. Numerical simulation of the hepatic circulation. International Journal of Artificial Organs. 27(3):222-230.

[34] Cohen RD, Brown CL, Nickols C, Levey P, Boucher BJ, Greenwald SE, and Wang W. 2011. Inbuilt mechanisms for overcoming functional problems inherent in hepatic microlobular structure. Comput. Math. Method Med. 2011:185845.

[35] Ekataksin W. 2000. The isolated artery: an intrahepatic arterial pathway that can bypass the lobular parenchyma in mammalian livers. Hepatology. 31:269-279.

[36] Ekataksin W, and Wake K. 1997. New concepts in biliary and vascular anatomy of the liver. Progress in Liver Diseases 15:1-30.

[37] Barbee JH, and Cokelet GR. 1971. The Fahraeus effect. Microvascular Research. 3(1):6-16. 
Table 1. Overview of all simulations with the corresponding VS and permeability conditions, as well as the longitudinal pressure gradient, the lobule circumferential diameter, the pressure drop between the PTs and CV, the VS thickness, and the PT and CV diameters.

\begin{tabular}{|c|c|c|c|c|c|c|c|c|}
\hline Model & 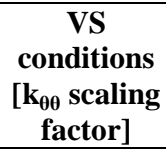 & $\begin{array}{c}\text { Permeability } \\
\text { conditions }\end{array}$ & $\begin{array}{c}\text { Pressure } \\
\text { gradient (z) } \\
{[\mathbf{m m H g} / \mathbf{m m}]}\end{array}$ & $\begin{array}{c}\text { Lobule } \\
\text { diameter } \\
{[\mathrm{mm}]}\end{array}$ & $\begin{array}{l}\text { PT-CV pressure } \\
\text { difference }[\mathrm{mmHg}]\end{array}$ & $\begin{array}{c}\text { VS thickness } \\
{[\mu \mathrm{m}]}\end{array}$ & $\begin{array}{c}\text { PT } \\
\text { diameter } \\
{[\mu \mathrm{m}]}\end{array}$ & $\begin{array}{c}\mathrm{CV} \\
\underset{[\mu \mathrm{m}]}{\operatorname{diameter}}\end{array}$ \\
\hline M0I & 1 & Isotropic & 1 & 1 & 0.95 & 10 & 40 & 60 \\
\hline MOA & 1 & Anisotropic & 1 & 1 & 0.95 & 10 & 40 & 60 \\
\hline M10I & 10 & Isotropic & 1 & 1 & 0.95 & 10 & 40 & 60 \\
\hline M10A & 10 & Anisotropic & 1 & 1 & 0.95 & 10 & 40 & 60 \\
\hline M100I & 100 & Isotropic & 1 & 1 & 0.95 & 10 & 40 & 60 \\
\hline M100A & 100 & Anisotropic & 1 & 1 & 0.95 & 10 & 40 & 60 \\
\hline M100A_1 & 100 & Anisotropic & 0 & 1 & 0.95 & 10 & 40 & 60 \\
\hline M100A_2 & 100 & Anisotropic & 0.5 & 1 & 0.95 & 10 & 40 & 60 \\
\hline M100A_3 & 100 & Anisotropic & 2 & 1 & 0.95 & 10 & 40 & 60 \\
\hline M100A_4 & 100 & Anisotropic & 1 & 1 & 1.45 & 10 & 40 & 60 \\
\hline M100A_5 & 100 & Anisotropic & 1 & 1 & 1.95 & 10 & 40 & 60 \\
\hline M100A_6 & 100 & Anisotropic & 1 & 1 & 0.95 & 5 & 40 & 60 \\
\hline M100A_7 & 100 & Anisotropic & 1 & 1 & 0.95 & 10 & 60 & 60 \\
\hline M100A_8 & 100 & Anisotropic & 1 & 1 & 0.95 & 10 & 40 & 90 \\
\hline M100A_9 & 100 & Anisotropic & 1 & 0.5 & 0.95 & 10 & 40 & 60 \\
\hline M100A_10 & 100 & Anisotropic & 1 & 0.5 & 1.45 & 10 & 40 & 60 \\
\hline M100A_11 & 100 & Anisotropic & 1 & 0.5 & 1.95 & 10 & 40 & 60 \\
\hline
\end{tabular}


Table 2. Overview of results for the simulated cases: the lobule and estimated total liver flow (Q) and resistance (R; see Appendix for details), as well as the percentage of difference of the lobule and total liver outflow relative to the baseline model M100A (calculated as $\left.\left(\mathrm{Q}_{\mathrm{Mx}}-\mathrm{Q}_{\mathrm{M} 100 \mathrm{~A}}\right) / \mathrm{Q}_{\mathrm{M} 100 \mathrm{~A}}\right)$.

\begin{tabular}{|c|c|c|c|c|c|c|}
\hline Model & $\begin{array}{c}\text { Q }_{\text {lobule }} \\
{[\mathrm{ml} / \mathrm{min}]}\end{array}$ & $\begin{array}{c}\text { Q }_{\text {liver }} \\
{[\mathrm{ml} / \mathrm{min}]}\end{array}$ & $\begin{array}{c}\text { R}_{\text {lobule }} \\
{[\mathrm{mmHg} \cdot \mathbf{m i n} /} \\
\mathrm{ml}]\end{array}$ & $\begin{array}{c}\mathbf{R}_{\text {liver }} \\
{[\mathrm{mmHg} \cdot} \\
\mathrm{min} / \mathrm{ml}]\end{array}$ & $\begin{array}{c}\text { Percentual } \\
\text { difference } \\
\text { of } Q_{\text {lobule }} \\
\end{array}$ & $\begin{array}{c}\text { Percentual } \\
\text { difference } \\
\text { of } Q_{\text {liver }} \\
\end{array}$ \\
\hline MOI & $5.95 \cdot 10^{-5}$ & $1.37 \cdot 10^{2}$ & $1.60 \cdot 10^{4}$ & $6.91 \cdot 10^{-3}$ & $-12.2 \%$ & $-12.2 \%$ \\
\hline M0A & $6.02 \cdot 10^{-5}$ & $1.39 \cdot 10^{2}$ & $1.58 \cdot 10^{4}$ & $6.83 \cdot 10^{-3}$ & $-11.2 \%$ & $-11.2 \%$ \\
\hline M10I & $6.54 \cdot 10^{-5}$ & $1.51 \cdot 10^{2}$ & $1.45 \cdot 10^{4}$ & $6.29 \cdot 10^{-3}$ & $-3.6 \%$ & $-3.6 \%$ \\
\hline M10A & $6.58 \cdot 10^{-5}$ & $1.52 \cdot 10^{2}$ & $1.44 \cdot 10^{4}$ & $6.26 \cdot 10^{-3}$ & $-3.0 \%$ & $-3.0 \%$ \\
\hline M100I & $6.77 \cdot 10^{-5}$ & $1.57 \cdot 10^{2}$ & $1.40 \cdot 10^{4}$ & $6.08 \cdot 10^{-3}$ & $-0.1 \%$ & $-0.1 \%$ \\
\hline M100A & $6.78 \cdot 10^{-5}$ & $1.57 \cdot 10^{2}$ & $1.40 \cdot 10^{4}$ & $6.07 \cdot 10^{-3}$ & $\begin{array}{c}0.0 \% \\
\text { (baseline) }\end{array}$ & $\begin{array}{c}0.0 \% \\
\text { (baseline) }\end{array}$ \\
\hline M100A_1 & $6.78 \cdot 10^{-5}$ & $1.57 \cdot 10^{2}$ & $1.40 \cdot 10^{4}$ & $6.07 \cdot 10^{-3}$ & $0.0 \%$ & $0.0 \%$ \\
\hline M100A_2 & $6.78 \cdot 10^{-5}$ & $1.57 \cdot 10^{2}$ & $1.40 \cdot 10^{4}$ & $6.07 \cdot 10^{-3}$ & $0.0 \%$ & $0.0 \%$ \\
\hline M100A_3 & $6.78 \cdot 10^{-5}$ & $1.57 \cdot 10^{2}$ & $1.40 \cdot 10^{4}$ & $6.07 \cdot 10^{-3}$ & $0.0 \%$ & $0.0 \%$ \\
\hline M100A_4 & $1.03 \cdot 10^{-4}$ & $2.39 \cdot 10^{2}$ & $1.40 \cdot 10^{4}$ & $6.07 \cdot 10^{-3}$ & $52.6 \%$ & $52.6 \%$ \\
\hline M100A_5 & $1.39 \cdot 10^{-4}$ & $3.21 \cdot 10^{2}$ & $1.40 \cdot 10^{4}$ & $6.07 \cdot 10^{-3}$ & $105.2 \%$ & $105.2 \%$ \\
\hline M100A_6 & $6.51 \cdot 10^{-5}$ & $1.50 \cdot 10^{2}$ & $1.46 \cdot 10^{4}$ & $6.32 \cdot 10^{-3}$ & $-4.0 \%$ & $-4.0 \%$ \\
\hline M100A_7 & $7.01 \cdot 10^{-5}$ & $1.62 \cdot 10^{2}$ & $1.35 \cdot 10^{4}$ & $5.87 \cdot 10^{-3}$ & $3.4 \%$ & $3.4 \%$ \\
\hline M100A_8 & $7.80 \cdot 10^{-5}$ & $1.80 \cdot 10^{2}$ & $1.22 \cdot 10^{4}$ & $5.27 \cdot 10^{-3}$ & $15.1 \%$ & $15.1 \%$ \\
\hline M100A_9 & $9.74 \cdot 10^{-5}$ & $9.00 \cdot 10^{2}$ & $9.75 \cdot 10^{3}$ & $1.06 \cdot 10^{-3}$ & $43.7 \%$ & $474.8 \%$ \\
\hline M100A_10 & $1.49 \cdot 10^{-4}$ & $1.37 \cdot 10^{3}$ & $9.75 \cdot 10^{3}$ & $1.06 \cdot 10^{-3}$ & $119.3 \%$ & $777.4 \%$ \\
\hline M100A_11 & $2.00 \cdot 10^{-4}$ & $1.85 \cdot 10^{3}$ & $9.75 \cdot 10^{3}$ & $1.06 \cdot 10^{-3}$ & $195.0 \%$ & $1079.9 \%$ \\
\hline
\end{tabular}


Figure 1. Schematic representation of the hepatic microcirculation. (a) Liver lobule with the shape of a hexagonal prism and its vascular network; (b) Spatial organization of the liver lobules. A liver acinus is indicated by the white dotted line.

(a)

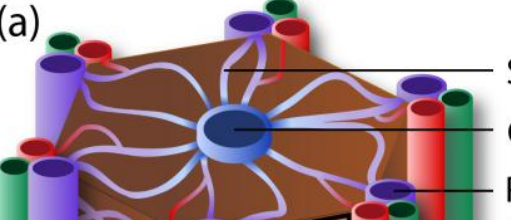

Sinusoids

Central vein

Portal venule

Hepatic arteriole

Bile ductule

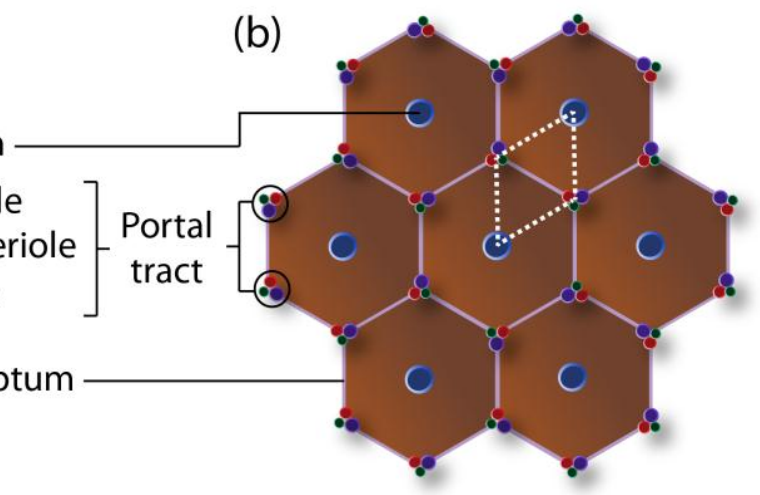


Figure 2. (a) Simulation geometry of a liver lobule; (b) pressure contours (in $\mathrm{mmHg}$ ) obtained in model M100A. The $z$-axis of the cylindrical coordinate system is located along the longitudinal axis of the lobule and its origin is at the centre of the simulation geometry.

(a) Lobule geometry

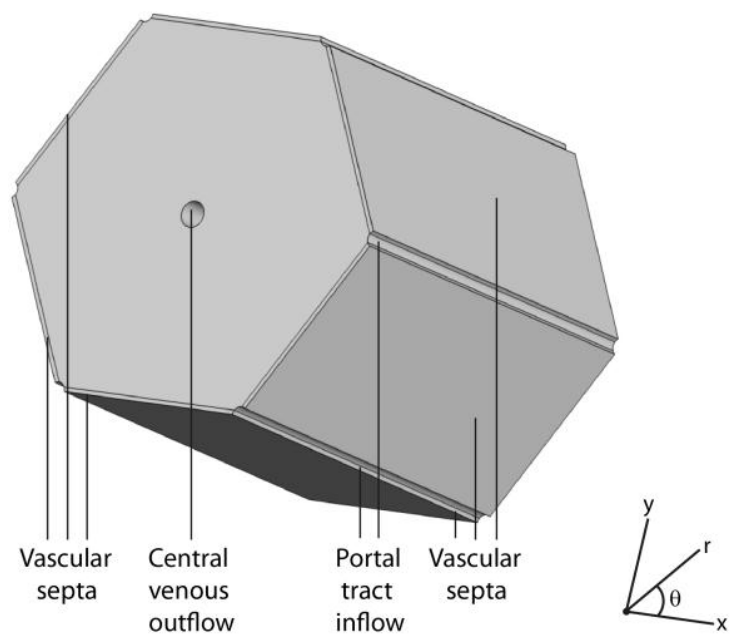

(b) Pressure contours of lobule model M100A

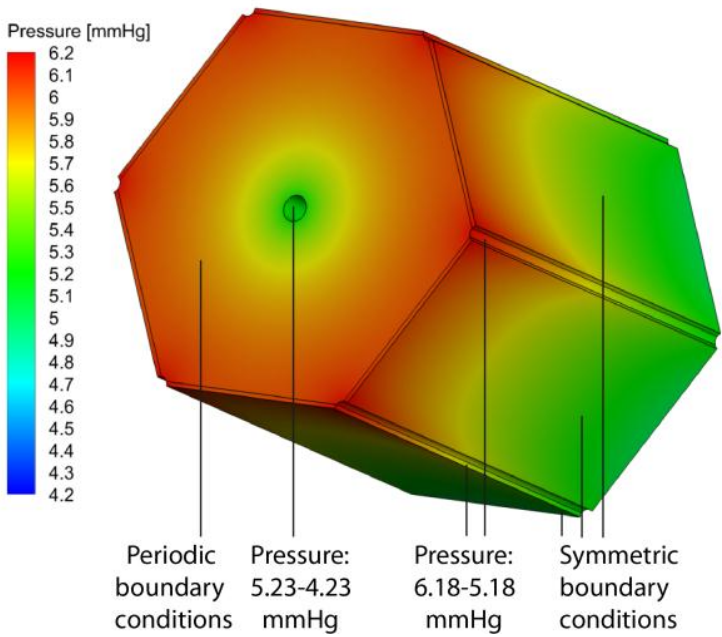


Figure 3. Comparison of the pressures and velocities at particular locations in the lobule for the models M0I, M0A, M10I, M10A, M100I and M100A: (a) pressure at the points midway between neighbouring PTs; (b-d) magnitudes of the cross-sectional projection of the velocity $\left(\left\|\vec{v}_{r \theta}\right\|\right)$ : (b) at PT inlets (mean value over the PT inlet), (c) at the midpoints of the border lines of the sinusoidal volume connecting neighbouring PT, and (d) at the CV outlet (mean value over the CV outlet); (e-f) magnitude of the velocity projected into a longitudinal cross-section $\left(\left\|\vec{v}_{r z}\right\|\right)$ : (e) at PT inlets (longitudinal section through the PT, $\theta=0^{\circ}, 180^{\circ}$ ), and (f) at the VS inflow into the lobule (longitudinal section through the midpoints of the VS, $\theta= \pm 90^{\circ}$ ).
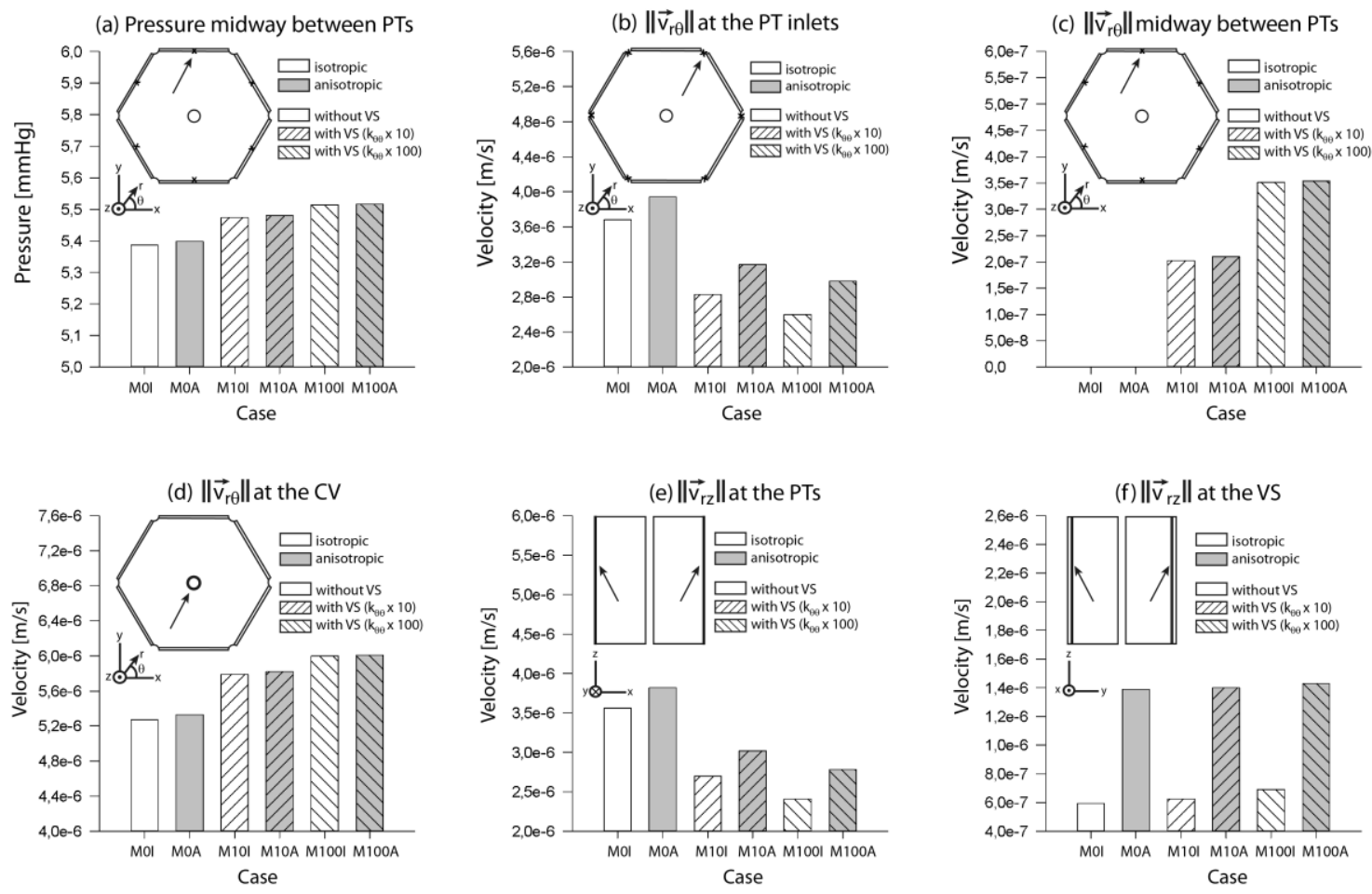
Figure 4. Pressures (top) and streamlines (bottom, coloured according to the magnitude of the projection of the velocity into the plane $\left.\left(\left\|\vec{v}_{r \theta}\right\|\right)\right)$ obtained on the middle crosssection ( $\mathrm{z}=0$ ) of the lobule models: (a) M0I, (b) M0A, (c) M10I, (d) M10A, (e) M100I, (f) M100A.
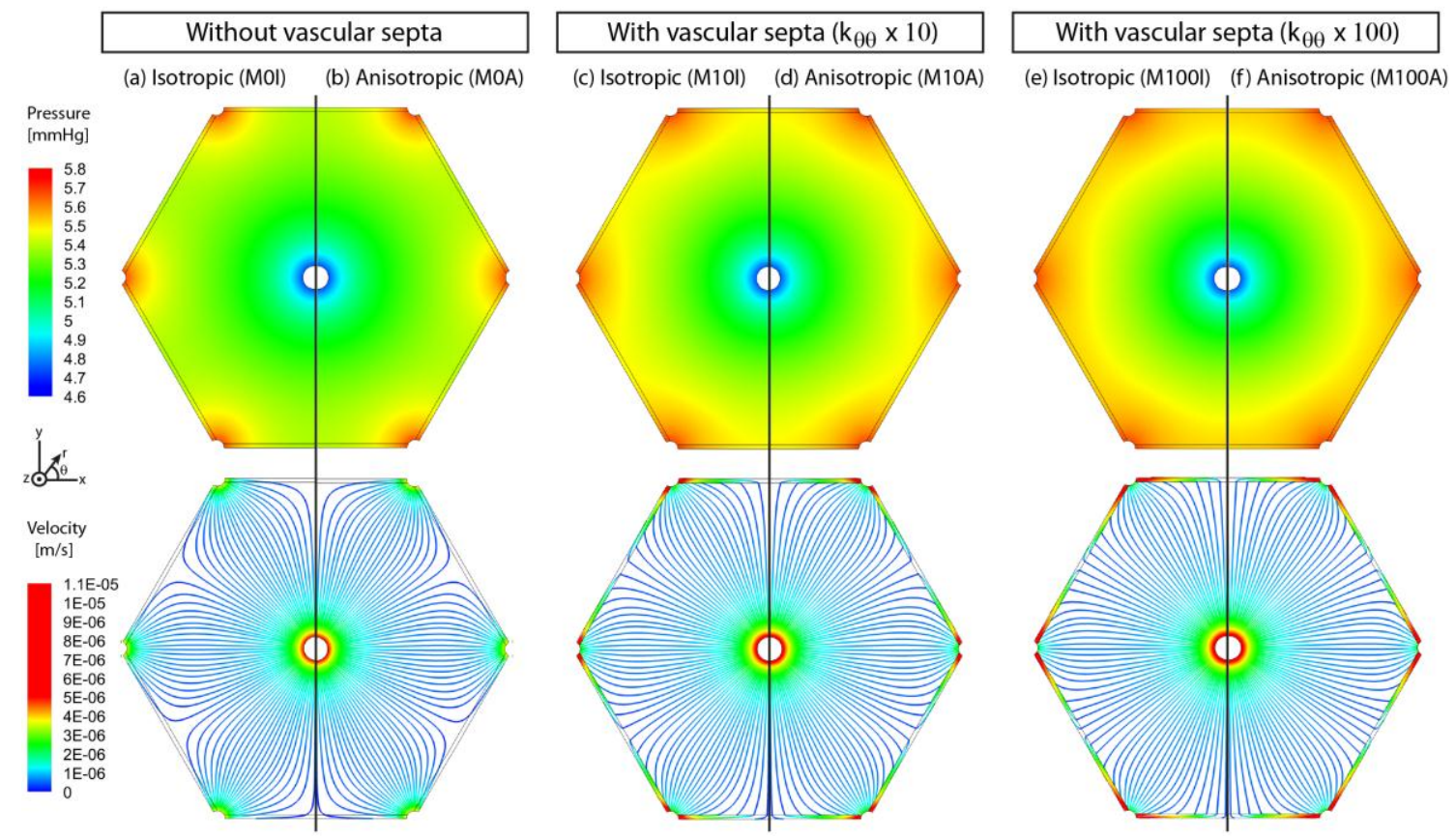
Figure 5. Pressures (top) and streamlines (bottom, coloured according to the magnitude of the projection of the velocity into the plane $\left.\left(\left\|\vec{v}_{r z}\right\|\right)\right)$ obtained on a longitudinal crosssection passing through two PTs $\left(\theta=0^{\circ}, 180^{\circ}\right)$ of the lobule models: (a) M0I, (b) M0A, (c) M10I, (d) M10A, (e) M100I, (f) M100A.

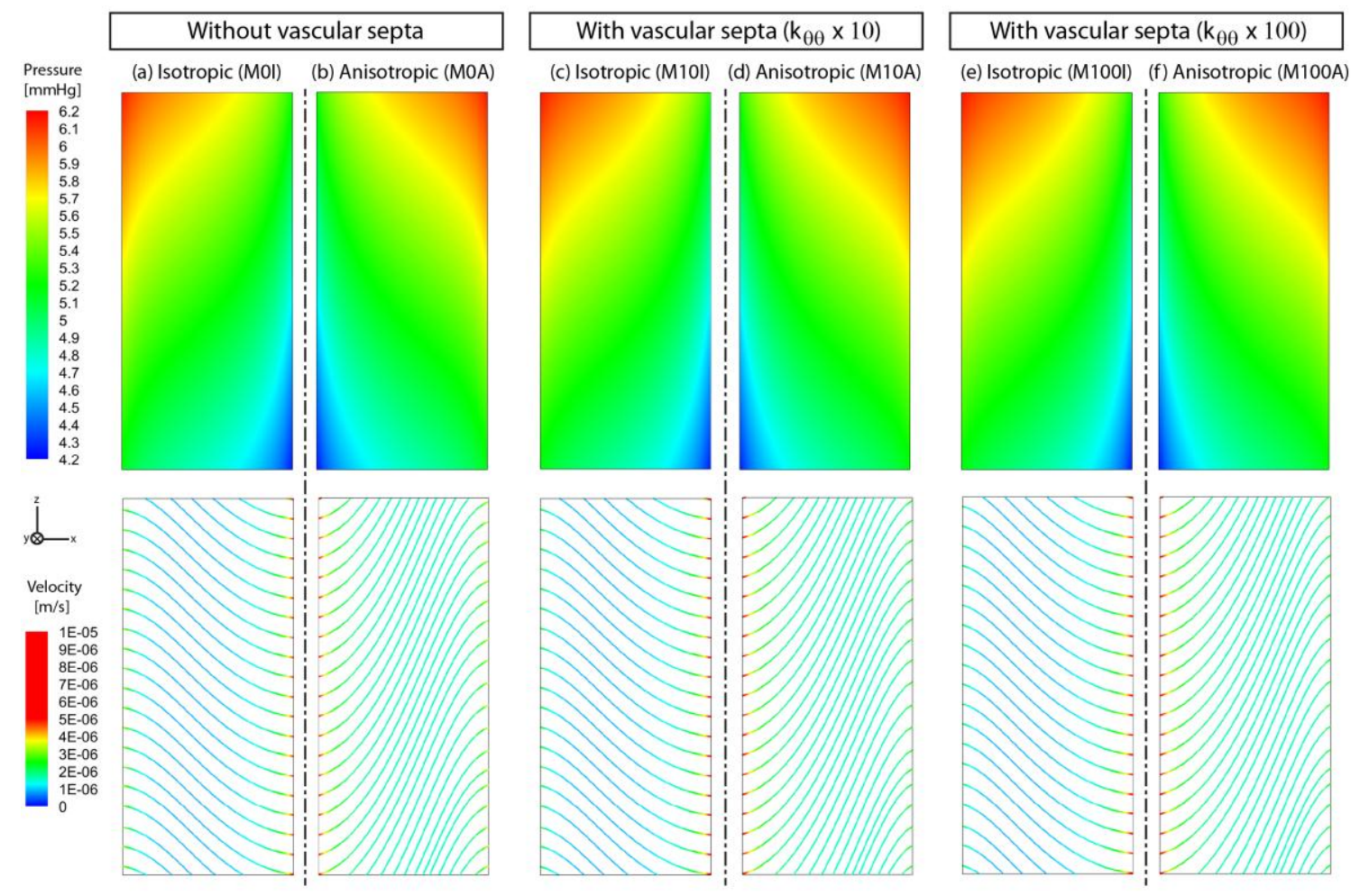


Figure 6. Pressures (top) and streamlines (bottom, coloured according to the magnitude of the projection of the velocity into the plane) obtained on a longitudinal cross-section passing through the centre of two opposite VSs $\left(\theta= \pm 90^{\circ}\right)$ of the lobule models: (a) M0I, (b) M0A, (c) M10I, (d) M10A, (e) M100I, (f) M100A.

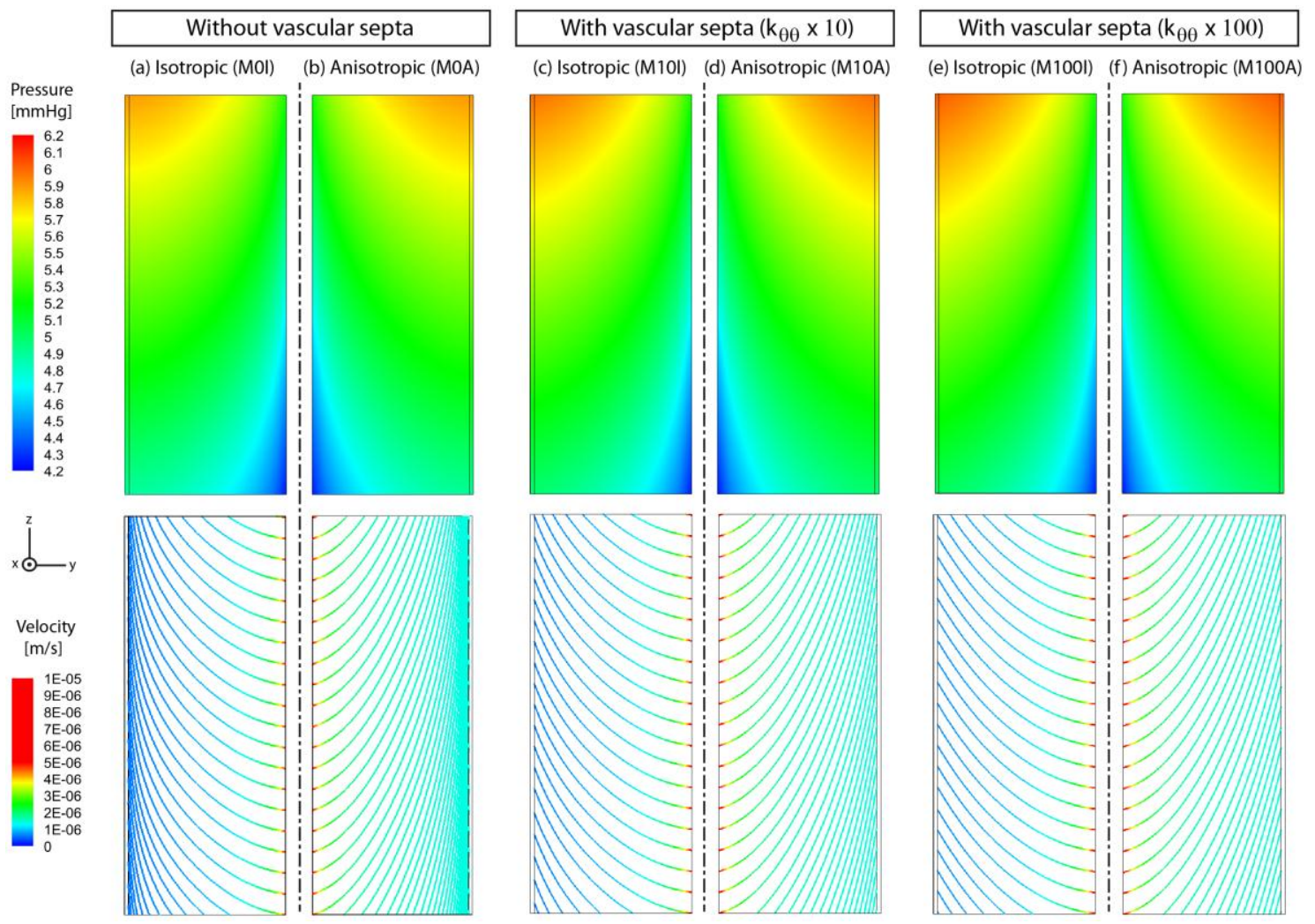


Figure 7. Comparison of the velocities at various locations for models M0A, M100I and M100A. (a-c) Magnitudes of the projection of the velocity in the cross-section $\left(\left\|\vec{v}_{r \theta}\right\|\right)$ : (a) along a line connecting the CV to the middle of a VS $\left(\theta=90^{\circ}\right.$ ) (the range of $r$ is $0.030 \mathrm{~mm}<r<0.433 \mathrm{~mm}$ ), (b) along a line connecting two PTs, and (c) around the circle centred on the $\mathrm{CV}$ with radius $0.3 \mathrm{~mm}$, plotted against the angle. (d) Magnitudes of the $3 \mathrm{D}$ velocity vector around the same circle as shown in (c).
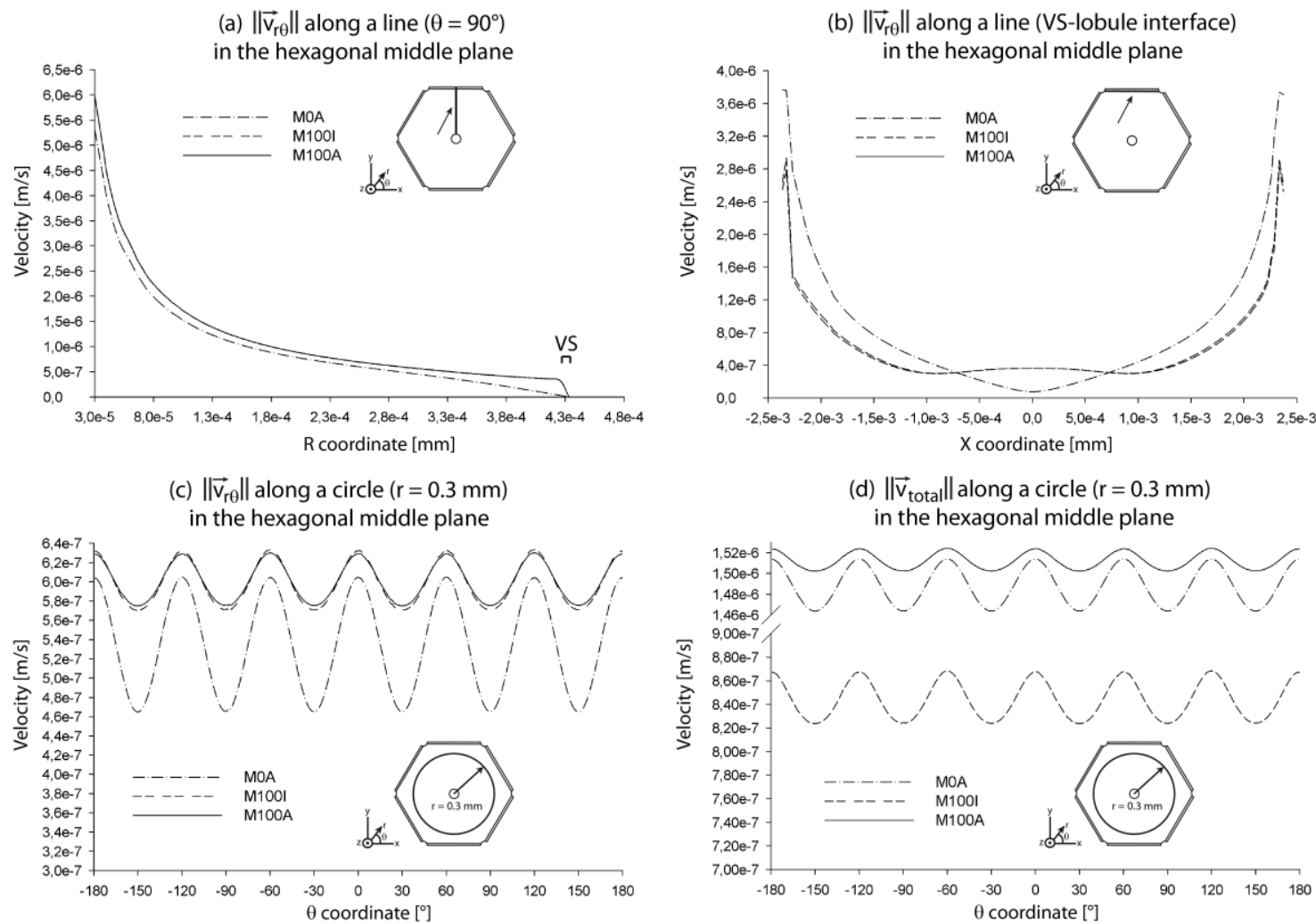
Figure 8. Pressures and streamlines (coloured according to the magnitude of the projection of the velocity into the plane $\left.\left\|\vec{v}_{r z}\right\|\right)$ obtained on a longitudinal cross-section passing through two PTs (top; $\theta=0^{\circ}, 180^{\circ}$ ) and on a longitudinal cross-section passing through the centre of two opposite VSs (bottom; $\theta= \pm 90^{\circ}$ ) for variations of the pressure gradient along the longitudinal $z$ direction $(0,0.5$ and $2 \mathrm{mmHg}$ corresponding to models M100A_1, M100A_2 and M100A_3, respectively) compared to the baseline model.

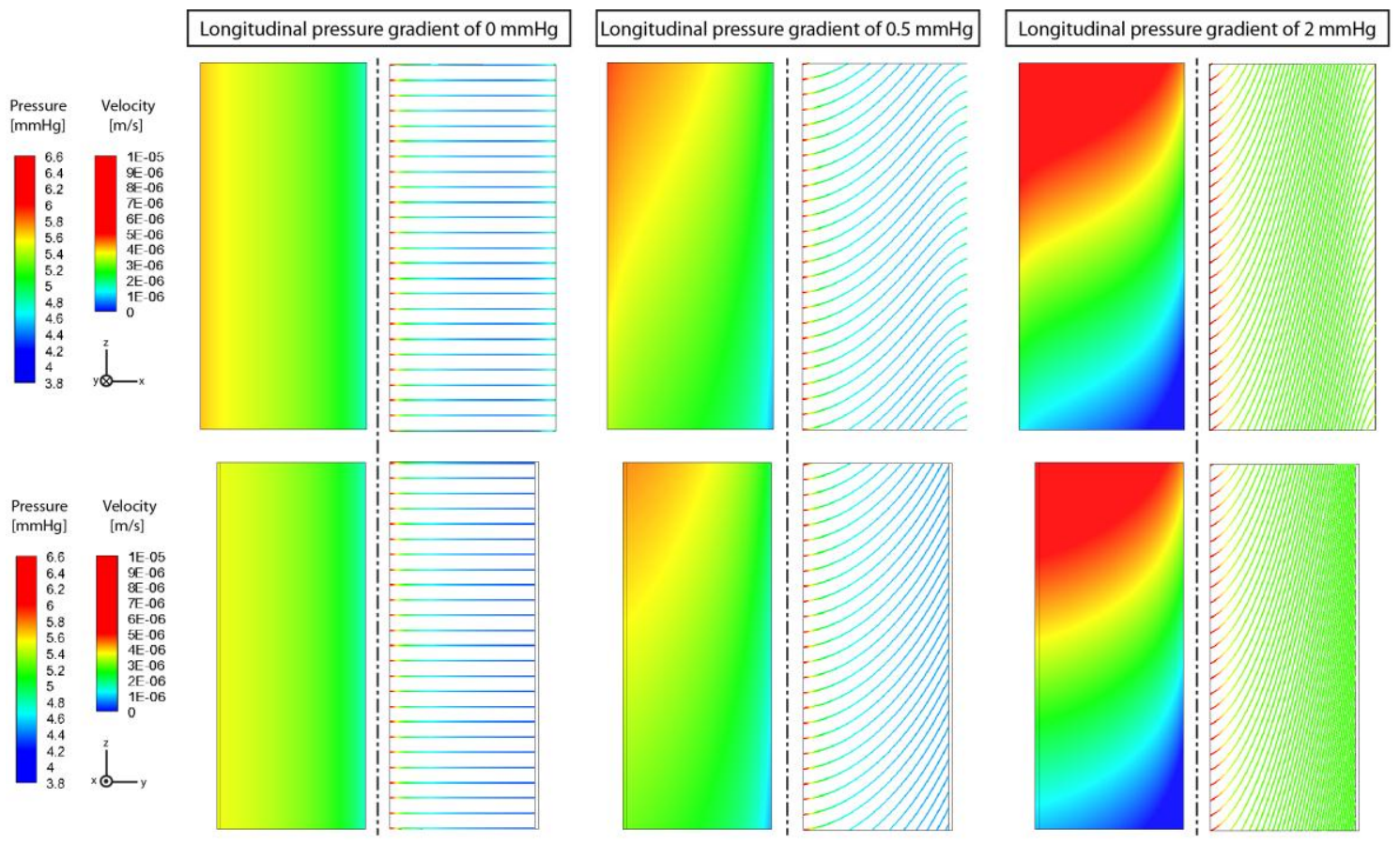


Figure 9. Pressures and streamlines (coloured according to the magnitude of the projection of the velocity into the plane $\left\|\vec{v}_{r \theta}\right\|$ ) obtained on the middle cross-section $(z=0)$ using a $0.5 \mathrm{mmHg}$ and $1 \mathrm{mmHg}$ increase of the PT pressure (corresponding to models M100A_4 and M100A_5, respectively) compared to the baseline model.

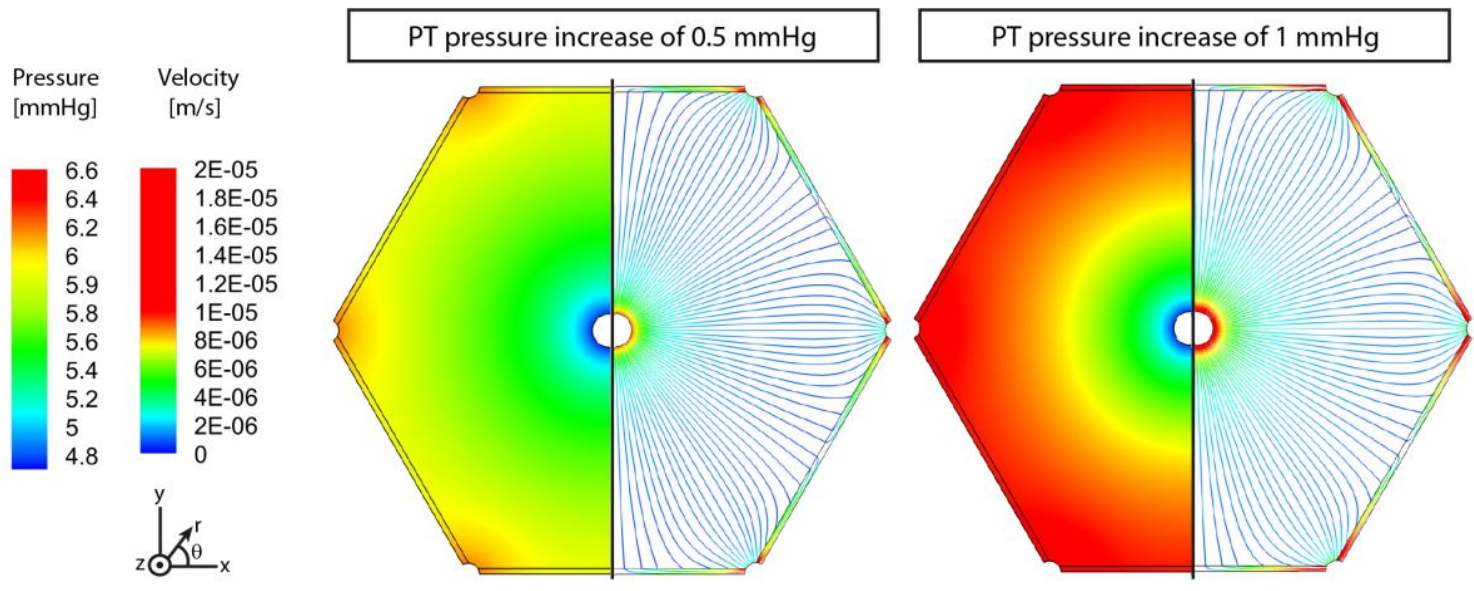


Figure 10. Pressures and streamlines (coloured according to the magnitude of the projection of the velocity into the plane $\left\|\vec{v}_{r \theta}\right\|$ ) obtained on the middle cross-section $(z=0)$ using variations of the geometrical features compared to the baseline model: VS thickness of $5 \mu \mathrm{m}$ (M100A_6), PT diameter of $60 \mu \mathrm{m}$ (M100A_7), CV diameter of 90 $\mu \mathrm{m}$ (M100A_8) and a lobule circumferential diameter of 0.5 mm (M100A_9).
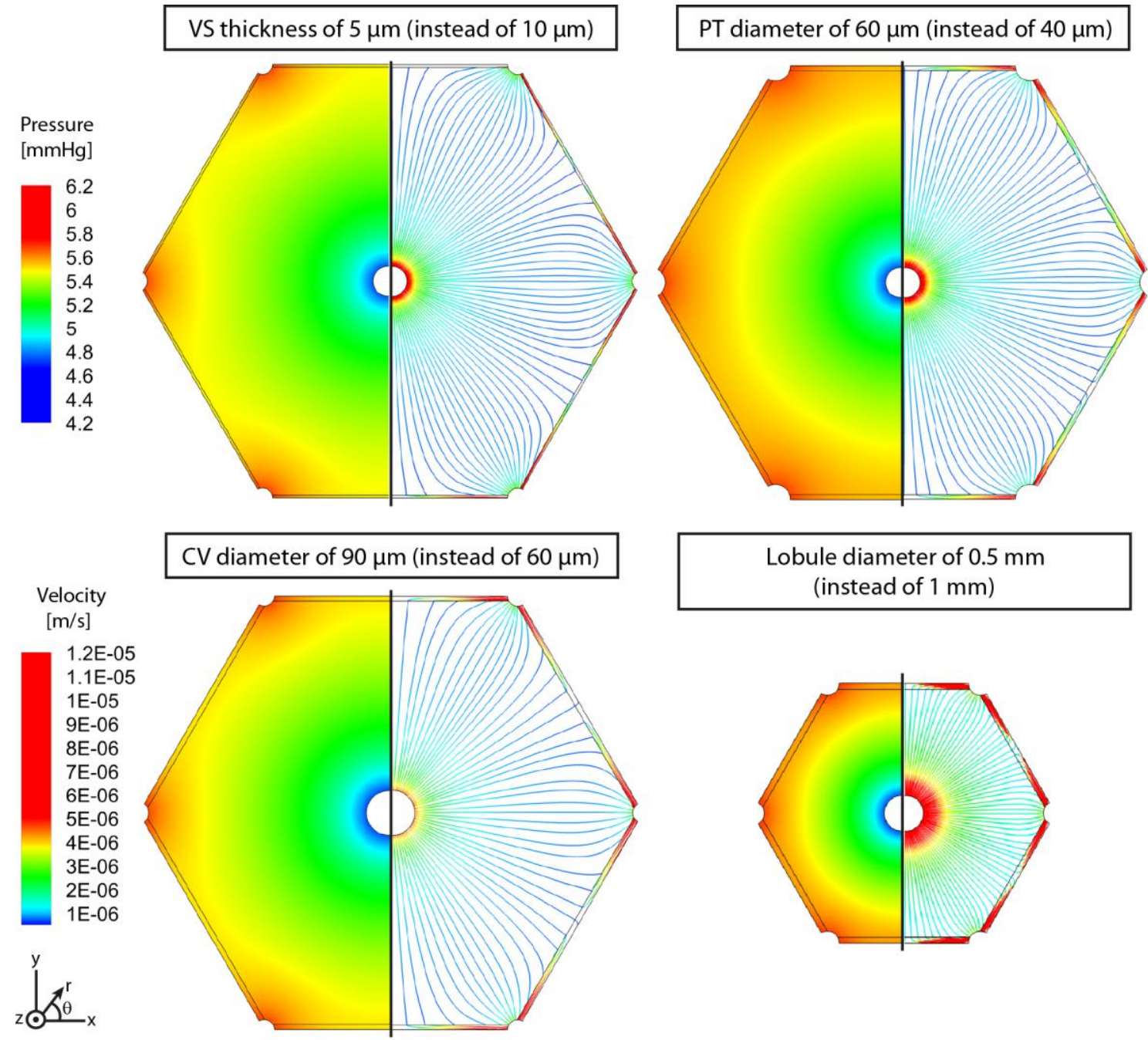